\title{
Light elements in the core: Effects of impurities on the phase diagram of iron
}

\author{
Alexander S. Côté, ${ }^{1}$ Lidunka Vočadlo, ${ }^{1}$ and John P. Brodholt ${ }^{1}$ \\ Received 25 November 2007; accepted 28 January 2008; published 11 March 2008.
}

[1] The stable phase of iron (Fe) in the Earth's core has been the subject of extensive studies, yet still remains controversial. Hexagonal close-packed structure (hcp) is the accepted stable form of pure Fe; however, it has been recently proposed that a body-centered-cubic (bcc) structured alloy of Fe with lighter elements would be a strong candidate. Experiments and ab initio calculations have shown that small amounts of silicon can stabilize the bcc phase with respect to the hep phase. In this study we examine the effect at core conditions by adding small concentrations of carbon (C), sulphur (S) and oxygen $(\mathrm{O})$ to iron using electronic structure calculations. We analyze the effect of the light elements on the energy difference between the two structures, and we conclude that the bcc phase tends to be stabilized by the alloys, strengthening the case for a bcc-structured inner core of the Earth. Citation: Côté, A. S., L. Vočadlo, and J. P. Brodholt (2008), Light elements in the core: Effects of impurities on the phase diagram of iron, Geophys. Res. Lett., 35, L05306, doi:10.1029/2007GL032788.

\section{Introduction}

[2] Determining the crystal structure of Earth's inner core would be a key to explaining the observed seismic anisotropy and layering [Morelli et al., 1986; Song and Helmberger, 1998; Yoshida, 1998; Ishii and Dziewonski, 2002], as well as the core's evolution. It is well known that the core is primarily composed of iron. Lower atomic weight elements have been suggested in smaller concentrations, to account for the measured density deficit [Birch, 1964], but their species, concentrations, and whether they have an influence on the core structure, remains an open question. Some of the candidates that have been proposed [Allègre et al., 2001] are silicon (Si), sulphur (S), carbon (C) and oxygen $(\mathrm{O})$.

[3] The crystal structure of iron in the inner core is an important factor for determining the light elements above and their composition; however its phase diagram at such extreme pressure and temperature conditions remains a matter of controversy. Recent theoretical studies [Vočadlo et al., 2003] showed that at core conditions, even though the hcp structure is marginally favored over bcc, the free energies of the bcc and hcp structures of pure iron are very similar. Furthermore, at low concentrations (7.9 weight \%), it was found that $\mathrm{Si}$ has a large effect on the stability field of bcc Fe, increasing the transition pressure to hcp from 10$15 \mathrm{GPa}$ up to $36 \mathrm{GPa}$ at $300 \mathrm{~K}$ [Lin et al., 2002; Côté et al.,

\footnotetext{
${ }^{1}$ Department of Earth Sciences, University College London, London, UK.
}

Copyright 2008 by the American Geophysical Union. 0094-8276/08/2007GL032788
2008]. Côté et al. argued that with an appropriate concentration of $\mathrm{Si}$ in the core the bcc phase would be entropically stabilized with respect to the hcp and the formation of a bccstructured Fe-Si alloy would be favored. Following that, we present here our $a b$ initio results on the effect of three other candidate light elements on the crystal structure of the inner core.

[4] In the next section we describe the simulation techniques used in our calculations and we explain how the equations of state for the different impurity concentrations in the different iron crystal structures were obtained, taking into account different possible substitutional configurations. We then show how the relative enthalpy curves can be derived, from which we deduce the stable structure at Earth's core pressures. Finally we discuss possible implications for the core's structure.

\section{Quantum Mechanical Simulation Techniques}

[5] The DFT calculations in this work were carried out using the VASP code [Kresse and Furthmüller, 1996]. The exchange-correlation energy was represented by the generalized gradient approximation (GGA) [Wang and Perdew, 1991], which achieves the highest accuracy for transition metals. Instead of pseudopotentials however, we made use of the projected augmented wave (PAW) method incorporated in VASP, where the wave function is not entirely pseudized, but combines an all-electron core region with a pseudo-valence region. The PAW method was introduced by Blöchl [1994], and it combines the accuracy of allelectron methods with the efficiency of pseudopotentials. With a relatively small number of plane waves, it can give results that agree accurately with all electron methods. Indeed, it has been previously shown that the use of this method gives accurate results for iron both under ambient and core conditions [Mao et al., 2001; Vočadlo et al., 2003; Alfè et al., 2001].

[6] Unit cells of pure iron were constructed in the bcc and hcp structures. For the bcc case we used the 2-atom ferromagnetic cubic unit cell. For the hcp case, the unit cell used was that of the anti-ferromagnetic II (afmII) structure which contains 8 atoms [Côté et al., 2008], as this was shown to be the stable hcp phase at low pressures [SteinleNeumann et al., 2004; Vočadlo et al., 2006]. We confirmed that at high pressures - above $\sim 40 \mathrm{GPa}$ - the magnetic structure disappears completely in hcp [Söderlind et al., 1996]. In bcc there is a small magnetic moment retained even at core pressures, however it was shown by Vočadlo et al. [2003] that it disappears at core temperatures.

[7] VASP calculates the ground state $(T=0)$ for each set of ionic positions and the electronic free energy is taken as the quantity to be minimized. Care was taken when choos- 
a)

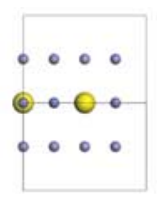

(i)

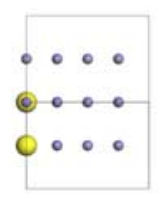

(ii)

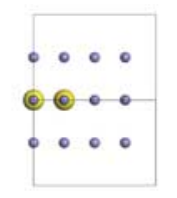

(iii)

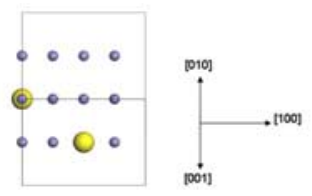

(iv)

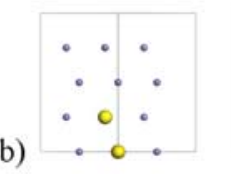

(i)

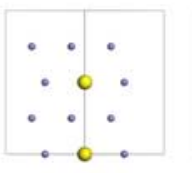

(ii)

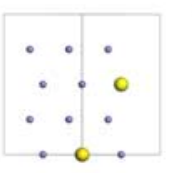

(iii)

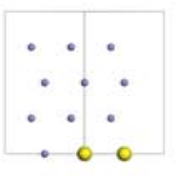

(iv)

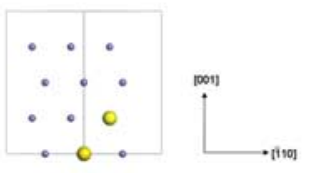

(v)

Figure 1. (a) The four different configurations of bcc viewed along $\langle 011\rangle$. Configuration (ii) is the most stable at high pressures. (b) The five different configurations of hcp viewed along $\langle 110\rangle$. Configuration (iii) is the most stable. The periodic boundary conditions are not shown in the pictures. The substituted atoms are enlarged for clarity.

ing the k-point sampling grid and cutoff energies in order to have an energy convergence of no more than $0.004 \mathrm{eV} /$ atom. A $14 \times 14 \times 14$ grid (equivalent to $84 \mathrm{k}$-points in the irreducible Brillouin zone (IBZ)) was used for bcc-Fe and a $9 \times 9 \times 9$ grid $(125 \mathrm{k}$-points in the IBZ) for hcp-Fe, with a cutoff energy of $400 \mathrm{eV}$. The same cutoff energy was used throughout the calculations.

[8] Iron supercells were then created, and substitutional defects of different light elements, namely S, C and $\mathrm{O}$ were incorporated at two different concentrations, $\sim 6.25$ and $\sim 12.5$ atm. \%. Specifically, for both structures, that meant a substitutional defect of one or two light element atoms respectively for each of the above concentrations. The supercell contained 16 atoms both in the bcc $($ a $2 \times 2 \times$ 2 multiplication of the 2-atom bcc unit cell) and in the hcp structure $($ a $1 \times 1 \times 2$ multiplication of the afm II unit cell). A $5 \times 5 \times 5 \mathrm{k}$-point grid (18 k-points in the IBZ) was used for the bcc and a $9 \times 9 \times 5(123 \mathrm{k}$-points in the IBZ) for the hcp phase. The structures were then allowed to relax and the total energy was calculated. For the 12.5 atm \% concentrations, where there were more than one light element defect, a number of trials were performed with all the different possible configurations of the defect atoms and the most favorable configuration at high pressure was chosen (Figure 1).

[9] Finding the ground state free energy, E, for a set of different volumes, $\mathrm{V}$, around the inner core boundary (ICB) pressure allowed us to fit the E-V data to a 3rd order BirchMurnaghan equation of state (EOS) of the following form:

$$
\begin{aligned}
E(V)= & \frac{9}{4} K_{0} V_{0} \frac{V_{0}}{V}\left[\frac{1}{2}\left(\frac{V_{0}}{V}\right)^{\frac{1}{3}}-\left(\frac{V}{V_{0}}\right)^{\frac{1}{3}}\right]+\frac{9}{16} K_{0}\left(K^{\prime}-4\right) V_{0}\left(\frac{V_{0}}{V}\right) \\
& \cdot\left[\left(\frac{V_{0}}{V}\right)-3\left(\frac{V_{0}}{V}\right)^{\frac{1}{3}}+3\left(\frac{V}{V_{0}}\right)^{\frac{1}{3}}\right]+E_{0}-\frac{9}{16} K_{0} V_{0} \\
\cdot & \left(K^{\prime}-6\right)
\end{aligned}
$$

[see, e.g., Poirier, 1991]. The resulting EOS parameters were then used to obtain the enthalpies $\mathrm{H}$, and to plot the enthalpy as a function of pressure for the bcc and hep phases of the different concentrations.

\section{Results}

[10] The simulation was focused at the pressure of the $\mathrm{ICB}$, that is around $330 \mathrm{GPa}$. Using the above technique, we calculated the difference in enthalpies, between the bcc and hcp structures of pure iron at first, and subsequently for two different concentrations of $\mathrm{S}, \mathrm{C}$ and $\mathrm{O}$. The results are illustrated in Figure 2, together with our previously found results for silicon [Côté et al., 2008] which include a higher concentration of $18.75 \mathrm{~atm} . \%$ (10.40 wt.\%). It can be seen that, even though at $0 \mathrm{~K} \mathrm{hcp} \mathrm{is} \mathrm{always} \mathrm{the} \mathrm{most} \mathrm{stable}$ phase, the enthalpy difference between the two phases for $\mathrm{S}$ and $\mathrm{Si}$ drops almost linearly with increasing light element concentration. Using that linear drop for silicon, it was deduced from the slope that the enthalpies of the Si defects are $2.5 \mathrm{eV}$ per defect atom more stable in the bcc structure than the hcp phase. This means that, for example, the suggested $\sim 5$ mol.\% (2.58 wt. \%) concentration of $\mathrm{Si}$ in the inner core [Stixrude et al., 1997; Lin et al., 2002; Vočadlo et al., 2003; Uchida et al., 2001; Fiquet et al., 2001; Badro et al., 2007] would stabilize the bcc structure by $125 \mathrm{meV}$. For $\mathrm{C}$ and $\mathrm{O}$, and particularly for the latter, the

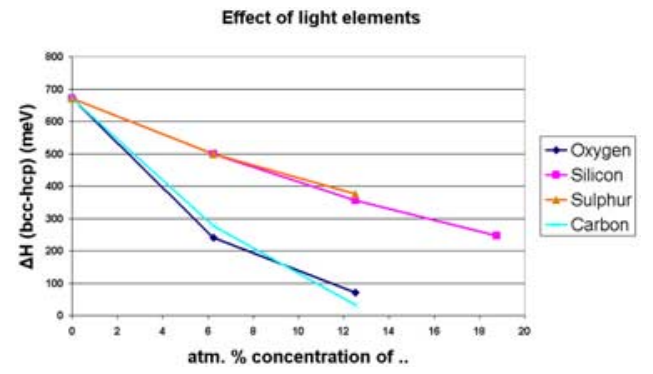

Figure 2. Light elements tend to stabilize bcc. The diagram shows the difference in enthalpy between the bcc and hep phase, which drops drastically with the addition of lighter elements to a pure iron crystal, reaching an order of magnitude less for a concentration of $12.5 \mathrm{~atm} \%$ of carbon and oxygen. 
drop in $\Delta H$ is clearly not linear; nevertheless it is clear from the graph that their effect towards stabilizing bcc is even greater than $\mathrm{S}$ and $\mathrm{Si}$, at least at small concentrations.

\section{Discussion and Conclusion}

[11] The above results, if combined with the previous results [Lin et al., 2002; Côté et al., 2008] on FeSi alloys at low pressure, leave no doubt that the inclusion of light elements tends to stabilize bcc-Fe. From Figure 2 it is apparent that our calculated enthalpy differences between the two structures at zero temperature are all positive, hence too large to favor bec stability at inner core pressures, at least for the suggested concentrations. However, it is suggested that the high temperatures of the core will alter this significantly. It has been recently shown that the difference between the free energies of the two phases of pure iron at the ICB is only $\sim 33 \mathrm{meV}$ [Vočadlo et al., 2003]. Since, as shown in our previous study [Côté et al., 2008], bcc is stabilized over hcp by at least $2.5 \mathrm{eV}$ per defect atom (for $\mathrm{Si}$ ), less than $1.5 \mathrm{~mol} \%$ of impurities would be needed to overcome the $33 \mathrm{meV}$ required to make bcc stable. The higher but still modest concentration of 5 mol.\% would provide a much larger stabilization enthalpy. This implies that even the suggested relatively small concentrations of lighter elements are more than enough to stabilize bcc-Fe at the inner core temperature and pressure.

[12] $\mathrm{Ab}$ initio calculations have shown that bcc iron is mechanically unstable at pressures above approximately $100 \mathrm{GPa}$ [Stixrude et al., 1994], but those calculations were performed at zero Kelvin. While Vočadlo et al. [2003] showed in their calculations that high temperatures act towards thermodynamically stabilizing the bcc phase, they did not consider the mechanical stability. This has been looked at recently (L. Vočadlo et al., The stability of bcc-Fe at high pressures and temperatures with respect to tetragonal strain, submitted to Physics of the Earth and Planetary Interiors, 2008) and they find that temperature does increase the mechanical stability of the bcc phase, and by $6000 \mathrm{~K}$, the bcc phase may indeed become dynamically stable. The inclusion of light elements may also have an added effect on the mechanical stability, and work is in progress towards that end.

[13] We conclude, therefore, that when considering the combined effects of temperature and light elements, the inner core is more likely to be in the bcc phase than in the hcp. Furthermore, recent work by Dubrovinsky [2007], showed that the bcc phase in the core is also stabilized by nickel; this further strengthens our conclusion. Confirming it would help us to understand the structure of the inner core, which would be a big step towards explaining the anisotropy and layering observed by seismology.

[14] Acknowledgments. The work of ASC was supported by NERC grant NE/C519662/1 and the Computational Mineral Physics consortium. We would also like to thank the Royal Society for the University Fellowship awarded to L.V.

\section{References}

Alfè, D., G. D. Price, and M. J. Gillan (2001), Thermodynamics of hexagonal-close-packed iron under Earth's core conditions, Phys. Rev. $B, 64,045123$.

Allègre, C., G. Manhès, and E. Lewin (2001), Chemical composition of the Earth and the volatility control on planetary genetics, Earth Planet. Sci. Lett., 185, 49-69.

Badro, J., G. Fiquet, F. Guyot, E. Gregoryanz, F. Occelli, D. Antonangeli, and M. d'Astuto (2007), Effect of light elements on the sound velocities in solid iron: Implications for the composition of Earth's core, Earth Planet. Sci. Lett., 254, 233-238.

Birch, F. (1964), Density and composition of mantle and core, J. Geophys. Res., 69, 4377-4388.

Blöchl, P. E. (1994), Projector augmented-wave method, Phys. Rev. B, 50, $17,953-17,979$.

Côté, A. S., L. Vočadlo, and J. P. Brodholt (2008), The effect of silicon impurities on the phase diagram of iron and possible implications for the Earth's core structure, J. Phys. Chem. Solids, in press.

Dubrovinsky, L. (2007), Body-centred cubic iron-nickel alloy in Earth's core, Science, 316, 1880-1883.

Fiquet, G., J. Badro, F. Guyot, H. Requardt, and M. Krisch (2001), Sound velocities in iron to 100 Gigapascals, Science, 291, 468-471.

Ishii, M., and A. M. Dziewonski (2002), The innermost inner core of the earth: Evidence for a change in anisotropic behavior at the radius of about 300 km, Proc. Natl. Acad. Sci. U. S. A., 99, 14,026-14,030.

Kresse, G., and J. Furthmüller (1996), Efficient iterative schemes for ab initio total-energy calculations using a plane-wave basis set, Phys. Rev. B, $54,11,169-11,186$.

Lin, J.-F., D. L. Heinz, A. J. Campbell, J. M. Devine, and G. Y. Shen (2002), Iron-silicon alloy in the Earth's core?, Science, 295, 313-315.

Mao, H. K., et al. (2001), Phonon density of states of iron up to 153 Gigapascals, Science, 292, 914-916.

Morelli, A., A. M. Dziewonski, and J. H. Woodhouse (1986), Anisotropy of the inner core inferred from PKIKP travel times, Geophys. Res. Lett., 13, $1545-1548$

Poirier, J. P. (1991), Introduction to the Physics of the Earth's Interior, pp. 66-74, Cambridge Univ. Press, Cambridge, U.K.

Söderlind, P., J. A. Moriarty, and J. M. Willis (1996), First-principles theory of iron up to Earth-core pressures: Structural, vibrational and elastic properties, Phys. Rev. B, 53, 14,063-14,072.

Song, X. D., and D. V. Helmberger (1998), Seismic evidence for an inner core transition zone, Science, 282, 924-927.

Steinle-Neumann, G., L. Stixrude, and R. E. Cohen (2004), Magnetism in dense hexagonal iron, Proc. Natl. Acad. Sci. U. S. A., 101, 33-36.

Stixrude, L., R. E. Cohen, and D. J. Singh (1994), Iron and high pressure: Linearized-augmented-plane-wave computations in the generalizedgradient approximation, Phys. Rev. B, 50, 6442-6445.

Stixrude, L., E. Wasserman, and R. E. Cohen (1997), Composition and temperature of Earth's inner core, J. Geophys. Res., 102, 24,729-24,739.

Uchida, T., Y. Wang, M. L. Rivers, and S. R. Sutton (2001), Stability field and thermal equation of state of e-iron determined by synchrotron X-ray diffraction in a multi-anvil apparatus, J. Geophys. Res., 106, 21,79921,810 .

Vočadlo, L., D. Alfè, M. J. Gillan, I. G. Wood, J. P. Brodholt, and G. D. Price (2003), Possible thermal and chemical stabilization of body centredcubic iron in the Earth's core, Nature, 424, 536-539.

Vočadlo, L., D. P. Dobson, and I. G. Wood (2006), An ab initio study of nickel substitution into iron, Earth Planet. Sci. Lett., 248, 132-137.

Wang, Y., and J. Perdew (1991), Correlation hole of the spin-polarized electron gas, with exact small-wave-vector and high-density scaling, Phys. Rev. B, 44, 13,298-13,307.

Yoshida, S. (1998), Models of the anisotropy of the Earth's inner core, J. Phys. Condensed Matter, 10, 11,215-11,226.

J. P. Brodholt, A. S. Côté, and L. Vočadlo, Department of Earth Sciences, University College London, Gower Street, London WC1E 6BT, UK. (a.cote@ucl.ac.uk) 